\title{
COMMENTARY
}

\section{Immunological monitoring to prevent and treat sepsis}

\author{
Raquel Almansa', John Wain², Eduardo Tamayo ${ }^{3}$, David Andaluz-Ojeda4, Ignacio Martin-Loeches 5 , Paula Ramirez \\ and Jesús F Bermejo-Martin*1
}

\begin{abstract}
The clinical, human and economic burden associated with sepsis is huge. Initiatives such as the Surviving Sepsis Campaign aim to effectively reduce risk of death from severe sepsis and septic shock. Nonetheless, although substantial benefits raised from the implementation of this campaign have been obtained, much work remains if we are to realise the full potential promised by this strategy. A deeper understanding of the processes leading to sepsis is necessary before we can design an effective suite of interventions. Dysregulation of the immune response to infection is acknowledged to contribute to the pathogenesis of the disease. Production of both proinflammatory and immunosuppressive cytokines is observed from the very first hours following diagnosis. In addition, hypogammaglobulinemia is often present in patients with septic shock. Moreover, levels of IgG, IgM and IgA at diagnosis correlate directly with survival. In turn, nonsurvivors have lower levels of C4 (a protein of the complement system) than the survivors. Natural killer cell counts and function also seem to have an important role in this disease. HLA-DR in the surface of monocytes and counts of $C D 4^{+} \mathrm{CD} 25^{+}$T-regulatory cells in blood could also be useful biomarkers for sepsis. At the genomic level, repression of networks corresponding to major histocompatibility complex antigen presentation is observed in septic shock. In consequence, cumulative evidence supports the potential role of immunological monitoring to guide measures to prevent or treat sepsis in a personalised and timely manner (early antibiotic administration, immunoglobulin replacement, immunomodulation). In conclusion, although diffuse and limited, current available information supports the development of large comprehensive studies aimed to urgently evaluate immunological monitoring as a tool to prevent sepsis, guide its treatment and, as a consequence, diminish the morbidity and mortality associated with this severe condition.
\end{abstract}

\section{Introduction}

The clinical, human and economic burden associated with sepsis is huge [1]. Initiatives such as the Surviving Sepsis Campaign [2], launched in 2002 as a collaborative initiative of the European Society of Intensive Care Medicine, the International Sepsis Forum, and the Society of Critical Care Medicine, aim to effectively reduce risk of death from severe sepsis and septic shock. Nonetheless, although substantial benefits raised from the implementation of this campaign have been obtained, much work remains if we are to realise the full potential promised by this strategy. Recently, new treatment approaches based on interventions for coagulation or inflammation have

*Correspondence: jfbermejo@saludcastillayleon.es

'Unidad de Investigación Biomédica del Clínico, Hospital Clínico Universitario de

Valladolid, SACYL/IECSCYL, Avda Ramón y Cajal 3, 47005 Valladolid, Spain

Full list of author information is available at the end of the article failed to improve survival in sepsis [1]. In addition, more effort needs to be made in the prevention of sepsis.

\section{Main text}

A deeper understanding of the processes leading to sepsis is necessary before we can design an effective suite of interventions [1]. Dysregulation of the immune response to infection is acknowledged to contribute to the pathogenesis of the disease [1]. Critical illness itself, surgery and concomitant comorbidities such as diabetes, chronic renal failure or chronic obstructive pulmonary disease affect host responses to infection [3,4], which could in turn facilitate the development of sepsis or impair outcome once sepsis is established.

Despite these precedents, the potential role of immunological monitoring in this disease has not been appropriately considered to the present moment. For years, two phases have been described in sepsis: an initial systemic inflammatory response syndrome followed by the negative feedback of a secondary compensatory 
anti-inflammatory response syndrome $[5,6]$. In contrast to this long-held view, Marchant and colleagues and our group have observed that production of the immunosuppressive cytokine IL-10 occurs from the very first hours following the diagnosis of severe sepsis or septic shock, and that it is directly associated with the secretion of proinflammatory cytokines [7-9]. In addition, hypogammaglobulinemia is often present in patients with septic shock [10]. Levels of IgG, IgM and IgA at diagnosis have been reported to correlate directly with survival $[11,12]$. In turn, nonsurvivors have lower levels of C4 (a protein of the complement system) than survivors [11]. Natural killer cell counts and function also seem to have an important role in this disease [11]. Severe depletion of immune effector cells is a universal finding in all age groups during sepsis [6]. Quantification of lymphocyte subsets and evaluation of their function could thus have diagnostic and prognostic value in sepsis. At the genomic level, repression of networks corresponding to major histocompatibility complex antigen presentation is observed in paediatric patients with septic shock [13].

As a consequence, cumulative evidence supports the notion that the immunological situation of the patient is linked to the final outcome in sepsis. Immunological monitoring could thus contribute to the prevention or the treatment of sepsis in a personalised and timely manner.

Interestingly, there is no currently available information on the potential role of proper immunological monitoring for the prevention of sepsis. Immunological monitoring could help to identify patients with immunological deficiencies (secondary to their disease process, treatment, and so forth) at higher risk for developing community acquired or nosocomial sepsis. Periodic monitoring of patients hospitalised in key services (that is, oncology, transplantation units, critical care units) could help to identify specific humoral or cellular immunity defects that could be addressed by implementation of prophylactic measures, such as administration of intravenous immunoglobulin (IVIG) or proper cover with broadspectrum antibiotics.

Prompt implementation of an accurate treatment is key to the final outcome in patients with sepsis [1]. Immunological monitoring could help to improve outcome in sepsis, by providing early detection of individuals at higher risk for developing complicated outcomes as well as relevant information for guiding treatment. For example, the treatment effect of IVIG on mortality for patients with septic shock is currently controversial. Measuring endogenous levels of immunoglobulins could improve the render of IVIG in sepsis, helping the clinician to better select those patients to be treated (those exhibiting marked hypogammaglobulinemia). Once more, there is a dramatic absence of information on the potential role of prior endogenous immunoglobulin quantification in the clinical assays evaluating IVIG for the treatment of this disease. Immunological monitoring could help also to guide therapies with immunomodulatory drugs with an anti-inflammatory effect or, alternatively, with an immunostimulatory effect [14].

Although not currently practiced, monitoring of the patient's immunological situation is feasible (at least from a quantitative point of view) in most hospitals. Quantification of immunoglobulins, complement proteins and $\mathrm{T}$ cells (CD4, CD8) in peripheral blood is an easily available routine test. Other tests of potential interest for immunological monitoring in sepsis include the quantification of HLA-DR in the surface of blood monocytes [15] or the evaluation of percentages of circulating $\mathrm{CD} 4{ }^{+} \mathrm{CD} 25^{+} \mathrm{T}$-regulatory cells in blood. Lastly, the use of genomic signatures (gene expression, miRNA, DNA methylation profiles) offers new opportunities to assess the immunological status of the patient. Combined evaluation of several immunological variables could provide added value over single ones.

\section{Conclusion}

Although diffuse and limited, current available information supports the development of large comprehensive studies aimed to urgently evaluate immunological monitoring as a tool to prevent or treat sepsis, and thereby to diminish the morbidity and mortality associated with this severe condition.

\section{Abbreviations \\ HLA, human leukocyte antigen; IVIG, intravenous immunoglobulin; IL, interleukin; miRNA, microRNA. \\ Competing interests \\ The authors declare that they have no competing interests. \\ Authors' contributions \\ RA, JW and JFB-M provided the immunological insight and participated in writing the article. ET, DA-O, IM-L and PR provided the clinical insight and participated in writing the article.}

\section{Acknowledgements}

The authors want to thank Dr David Livermore (Norwich Medical School, University of East Anglia) for his constructive comments on this letter.

\section{Author details}

Unidad de Investigación Biomédica del Clínico, Hospital Clínico Universitario de Valladolid, SACYL/ECSCYL, Avda Ramón y Cajal 3, 47005 Valladolid, Spain. ${ }^{2}$ Norwich Medical School, University of East Anglia. Norwich, NR4 7TJ, UK. ${ }^{3}$ Servicio de Anestesiología, Hospital Clínico Universitario de Valladolid, SACYL/ IECSCYL, Avda Ramón y Cajal 3, 47005 Valladolid, Spain. ${ }^{4}$ Servicio de Medicina Intensiva, Hospital Clínico Universitario de Valladolid, SACYL/IECSCYL, Avda Ramón y Cajal 3, 47005 Valladolid, Spain. ${ }^{5}$ Centro de Críticos, Corporación Sanitaria y Universitaria Parc Taulí - Hospital

de Sabadell, CIBER Enfermedades Infecciosas. Parc Taulí, 1. 08208 Sabadell, Spain. ${ }^{6}$ Servicio de Medicina Intensiva, Hospital Universitario y Politécnico la $\mathrm{Fe}$, Bulevar del Sur, 46026 Valencia, Spain.

Published: 25 January 2013 


\section{References}

1. For sepsis, the drugs don't work. Lancet Infect Dis 2012, 12:89.

2. Levy MM, Dellinger RP, Townsend SR, Linde-Zwirble WT, Marshall JC, Bion J, Schorr C, Artigas A, Ramsay G, Beale R, Parker MM, Gerlach H, Reinhart K, Silva E, Harvey M, Regan S, Angus DC: The Surviving Sepsis Campaign: results of an international guideline-based performance improvement program targeting severe sepsis. Intensive Care Med 2010, 36:222-231.

3. Monneret G, Venet F, Kullberg BJ, Netea MG: ICU-acquired immunosuppression and the risk for secondary fungal infections. Med Mycol 2011, 49(Suppl 1):S17-S23.

4. Kimura F, Shimizu H, Yoshidome $\mathrm{H}$, Ohtsuka M, Miyazaki M: Immunosuppression following surgical and traumatic injury. Surg Today 2010, 40:793-808.

5. Monneret G, Venet F, Pachot A, Lepape A: Monitoring immune dysfunctions in the septic patient: a new skin for the old ceremony. Mol Med 2008, 14:64-78.

6. Skrupky LP, Kerby PW, Hotchkiss RS: Advances in the management of sepsis and the understanding of key immunologic defects. Anesthesiology 2011, 115:1349-1362.

7. Marchant A, Deviere J, Byl B, De Groote D, Vincent JL, Goldman M: Interleukin-10 production during septicaemia. Lancet 1994, 343:707-708.

8. Tamayo E, Fernandez A, Almansa R, Carrasco E, Heredia M, Lajo C, Goncalves L, Gomez-Herreras Jl, de Lejarazu RO, Bermejo-Martin JF: Pro- and antiinflammatory responses are regulated simultaneously from the first moments of septic shock. Eur Cytokine Netw 2011, 22:82-87.

9. Andaluz-Ojeda D, Bobillo F, Iglesias V, Almansa R, Rico L, Gandia F, Resino S, Tamayo E, de Lejarazu RO, Bermejo-Martin JF: A combined score of pro- and anti-inflammatory interleukins improves mortality prediction in severe sepsis. Cytokine 2012, 57:332-336.
10. Venet F, Gebeile R, Bancel J, Guignant C, Poitevin-Later F, Malcus C, Lepape A Monneret G: Assessment of plasmatic immunoglobulin G, A and M levels in septic shock patients. Int Immunopharmaco/ 2011, 11:2086-2090.

11. Andaluz-Ojeda D, Iglesias V, Bobillo F, Almansa R, Rico L, Gandia F, Loma AM, Nieto C, Diego R, Ramos E, Nocito M, Resino S, Eiros JM, Tamayo E, de Lejarazu $\mathrm{RO}$, Bermejo-Martin JF: Early natural killer cell counts in blood predict mortality in severe sepsis. Crit Care 2011, 15:R243.

12. Tamayo E, Fernandez A, Almansa R, Carrasco E, Goncalves L, Heredia M, Andaluz-Ojeda D, March G, Rico L, Gomez-Herreras Jl, de Lejarazu RO, Bermejo-Martin JF: Beneficial role of endogenous immunoglobulin subclasses and isotypes in septic shock. J Crit Care 2012, 27:616-622.

13. Wong HR, Cvijanovich N, Allen GL, Lin R, Anas N, Meyer K, Freishtat RJ, Monaco M, Odoms K, Sakthivel B, Shanley TP; Genomics of Pediatric SIRS/ Septic Shock Investigators: Genomic expression profiling across the pediatric systemic inflammatory response syndrome, sepsis, and septic shock spectrum. Crit Care Med 2009, 37:1558-1566.

14. Leentjens J, Kox M, Koch RM, Preijers F, Joosten LA, van der Hoeven JG, Netea MG, Pickkers P: Reversal of immunoparalysis in humans in vivo: a doubleblind, placebo-controlled, randomized pilot study. Am J Respir Crit Care Med 2012, 186:838-845

15. Monneret $G$, Lepape A, Venet F: A dynamic view of mHLA-DR expression in management of severe septic patients. Crit Care 2011, 15:198.

doi:10.1186/cc11922

Cite this article as: Almansa $\mathrm{R}$, et al.: Immunological monitoring to prevent and treat sepsis. Critical Care 2013, 17:109. 